\title{
HTTP Adaptive Streaming on Improved LISP-MN Architecture
}

\author{
Musab Muhammad Isah* \\ College of Computer Science and Engineering, University of Hafar Al Batin, Saudi Arabia \\ Email:mmisah@uhb.edu.sa \\ *Corresponding Author: Musab Muhammad Isah, Email: mmisah@uhb.edu.sa
}

How to cite this paper: Musab Muhammad Isah (2021) HTTP Adaptive Streaming on Improved LISP-MN Architecture. Journal of the Institute of Electronics and Computer, 3 , 61-74.

https://doi.org/10.33969/JIEC.2021.31005

Received: August 31, 2021

Accepted: September 9, 2021

Published: September 10, 2021

Copyright $(2021$ by author(s) and Institute of Electronics and Computer. This work is licensed under the Creative Commons Attribution International License (CC BY 4.0).

http://creativecommons.org/licenses/by/4.0/

(c) (7) Open Access

\begin{abstract}
The increasing consumption of video content using mobile devices calls for a more efficient techniques of handling the enormous data sent and received across wireless Internet. The Loc/ID split architecture is a group of proposals geared towards enhancing the mobility of nodes on the Internet. Although Loc/ID protocols guarantee handover, they suffer excessive packet loss and long service disruption time during the process. In this paper, we posit that redirecting incoming packets to a nearby buffer node, while a handover is taking place, and forwarding the packets to the mobile device after it reconnects will reduce the negative impact of packet loss on user experience. Improvement in the performance of video services is one manifestation that could be observed. In line with this, we propose an improved architecture for one of the most popular Loc/ID protocols, Locator-Identifier Separation Protocol Mobile Node (LISP-MN) by adding a new node, termed loc-server, to LISP-MN components. Loc-server caches and forwards packets to a mobile device during its handover process. To evaluate the performance of the improved architecture, we implemented the system on a laboratory tested and developed a number of MPEG-DASH compliant players. The performance of the players streaming over the proposed architecture is then compared against vanilla LISP-MN architecture. Results of the evaluation show improved performance when running the players on top of the new architecture.
\end{abstract}

\section{Keywords}

Adaptive Bitrate Streaming, Loc/ID Split, LISP-MN, Mobility, MPEG DASH,Network Architecture

\section{Introduction}

Mobile devices are now the primary means of not only capturing and sharing video content but are also increasingly becoming the main tool for its consumption. More significantly, this trend is expected to grow in future. In fact, mobile data traffic is expected to continuously grow at the rate of 47\% annually between 2016-2021 [5], with video traffic accounting for three-fourths of it.

This new reality calls for more efficient techniques of handling the traversal of data packets across wireless networks as a mobile node (MN) moves between different points of attachment (PoA). One new approach is Locator-Identifier Separation Protocol Mobile 
Node (LISP-MN) [8]. LISP-MN is a member of a family of mobility management protocols called Locator-Identifier Split (Loc/ID) $[15,19]$ that decouple the two primary roles of an IP address: identifying and locating a node in a network. Within a LISP-MN network, an $\mathrm{MN}$ is equipped with an immutable endpoint identifier (EID) for node identification, and a routing locator (RLOC) to serve as a care-of address (CoA), which is allocated to, or configured by, the node on connecting to a new network. The MN creates data sessions with remote nodes using its EID. This ensures that the session is maintained regardless of the of MN's location. The RLOC, on the other hand, is used to route packets to the MN.

On the other hand, HTTP-based adaptive video streaming services (HAS) such as the MPEG Dynamic Adaptive Streaming over HTTP (MPEG-DASH or simply DASH) are becoming the de facto standard for video delivery. In HAS, video file is divided into chunks usually of equal temporal size. With each chunk encoded in multiple bitrates. Typically, a client continuously monitors and estimates its capabilities (e.g. available bandwidth), and then, based on the estimate, requests chunks with the highest sustainable video rate.

A typical DASH implementation directly or indirectly equates the available bandwidth with the average TCP throughput when making rate selection decisions. However, it is a well-known fact that TCP is sensitive to packet loss. It is shown in [4] that as little as $0.1 \%$ in packet loss can cause the TCP throughput to oscillate, and worst $0.5 \%$ loss can result in an up to $25 \%$ reduction in throughput [11]. Furthermore, it was shown in [13, 20] that when a mobile user streams a video using HAS service while relying on LISP-MN protocol ${ }^{1}$ clients experience long service disruption time (SDT) and excessive packet loss while moving between PoAs of independent networks. This was shown to have a severe impact on the performance of a typical HAS video player .

Our contribution in this paper is a new LISP-MN based scheme that helps reduces packet loss at the point of handover. For this, we enhanced the LISP-MN architecture with an addition of a new node we called loc-server. Loc-sever is tasked with caching the incoming traffic during handover and then forwarding it to the MN on the completion of the node's handover process. The performance of the proposed scheme is evaluated against the original LISP-MN protocol. To achieve this, we use MPEG-DASH compliant players. The rest of the paper is organised as follows. Section 2 presents the background works. In section 3, the architecture of the proposed scheme is introduced. Sections 4 and 5 evaluate and discuss the performance of the proposed scheme respectively, with section 6 focussing on the related work. Finally the paper is wrapped up with a conclusion in Section 7.

\section{Background}

\subsection{Locator Identifier Separation Protocol Mobile Node}

LISP-MN is a host-based mobility management protocol built on top of LISP [7]. Here, an $\mathrm{MN}$ is equipped with a lightweight version of ingress/egress tunnel router (ITR/ETR) functionality of LISP, and behaves as a single domain. The MN is configured statically with an EID (the host identifier), which is used by the transport and upper layers of the OSI network model to identify communication sessions. The MN also configures CoA, termed RLOC, used in determining the MN's location. A map-server is a component of the LISP mapping system serving as the mobility anchor and tracks the location of the $\mathrm{MN}$ at any given time by caching the EID-to-RLOC mapping. LISP-MN specifies the need to use proxy ETR (PETR) to send packets and proxy ITR (PITR) to receive from networks with no LISP capability. The proxy functionality is usually collocated on a network node termed PXTR.

Upon attachment to a network, an MN configures a new RLOC and sends a map-register message to its map server in order to register the RLOC (its location), as shown in Fig. 1A. The server will authenticate the EID in the packet and reply with a map-notify message

\footnotetext{
${ }^{1}$ The study shows that a similar behaviour was observed when MIPv6 and PMIv6 mobility management protocols were used.
} 


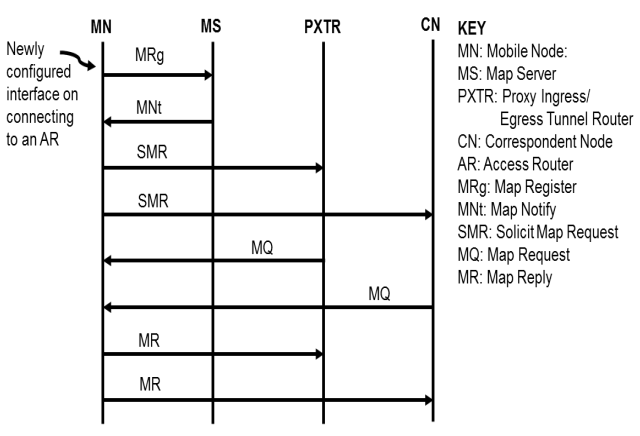

A

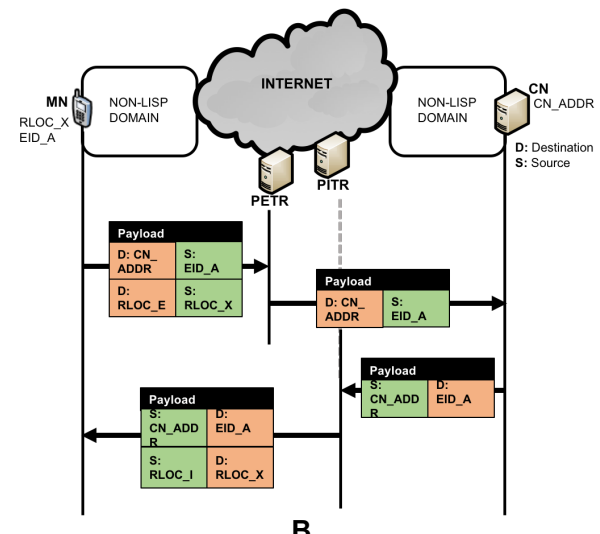

B

Figure 1. Vanilla LISP-MN: Control Plane (A) and Data Plane (B) Operations.

confirming that the EID-RLOC registration has been successful. The MN will also send a solicit-map-request (SMR) message to its PITR (PXTR in Fig. 1A), and to any LISP-based correspondent node $(\mathrm{CN})^{2}$ to cause the two nodes to request for the MN's new location. Consequently, the PITR and the $\mathrm{CN}$ send a map-request to the $\mathrm{MN}$, to which the MN replies with a map-reply containing the mobile device's new RLOC and its EID.

As shown in Fig. 1B, an MN encapsulates outgoing packets to the PETR, and the proxy router de-encapsulates the packets and forwards using the conventional Internet routing system. PETR will have the sending domain's EID-prefix pre-configured in its database for the router to serve nodes in that domain. Replies are sent by the CN using the MN's EID but are delivered by the Internet routing infrastructure to the PITR serving the EID, which then encapsulates and forwards the packets to the $\mathrm{MN}$.

\subsection{HTTP-based Adaptive Streaming}

HTTP-based adaptive video streaming services start by fragmenting video file into temporally equal size. Then, each fragment, called a chunk, is encoded into several bitrates. The chunks are stored together with a meta data file called Media Presentation Protocol(MPD)[1]. To stream a particular video, a client begins by requesting the MPD file from the server, typically, using a GET request. After which the client monitors and estimates the capability of its context, e.g. the available throughput and buffer level. A client progressively requests the next chunk, the bitrate of each video chunk requested is determined by client's measurement of its available resources. The HAS module that decides which bitrate is selected is called Adaptive Bitrate Selection(ABR). The ABR logic that solely rely on throughput estimation for video rate selection are called throughput-based [2], while those that rely on only buffer occupancy are called buffer-based [12, 21]. In this paper, we call the HAS players that use both throughput estimation and buffer occupancy in video rate selection, mixed-mode [17].

\section{Improved LISP-MN Architecture}

To improve the performance of LISP-MN, a loc-server is proposed. Loc-server is an additional component to the LISP-MN architecture, which serves as a buffer node. The new node serves as a support entity for packet delivery to ensure that no packet is lost as a result of the mobility process. MNs are equipped with the anycast address of loc-servers provided by the devices' domain (home network) and an MN will use the server that is

\footnotetext{
${ }^{2}$ These signals are not sent to non-LISP CN
} 


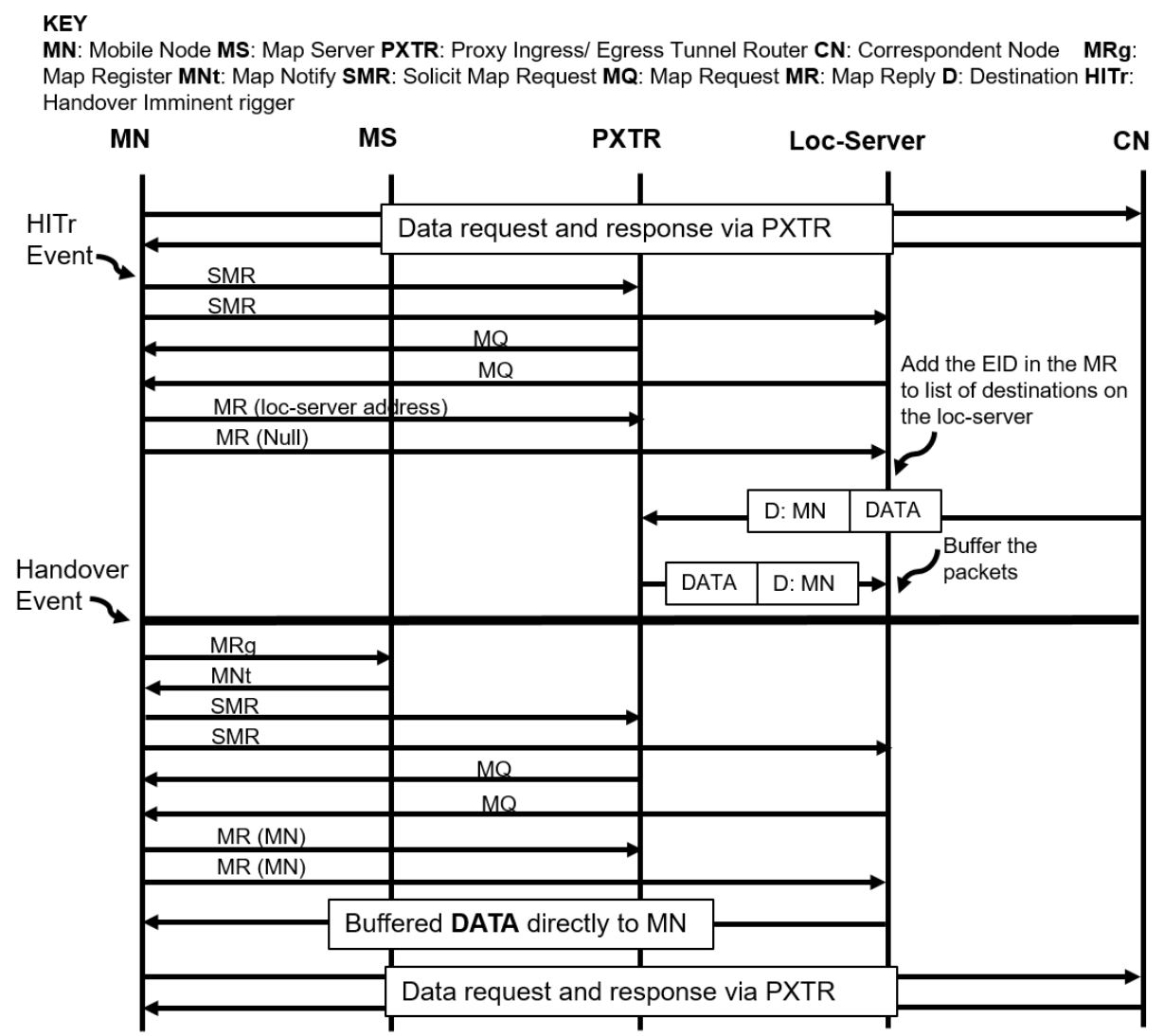

Figure 2. Improved LISP-MN: Control Plane and Data Plane Operations.

closest to its current location. Fig. 2 depicts the interaction between the MN, the PXTR and the loc-server in both the control plane and the data plane modes. We discussed the newly developed Improved LISP-MN architecture in detail in [14].

In the vanilla LISP-MN architecture, updates are only triggered when an MN's interface is (re)configured either due to a handover or when the MN has just come online. The MN indicates to the map-server and the PXTR of its new location by sending map-register and SMR to the two remote nodes respectively. In the improved LISP-MN architecture, however, mapping updates are also sent on the brink of a handover for the MN to tell the PXTR and the loc-server of its movement. A new handover imminent trigger (HITr) module is introduced on the MNs to inform the LISP-MN protocol when the signal strength of the current link (or other defined handover metrics such as cost of link usage, power consumption, quality of service, etc) has reached a defined threshold, which then prompt the protocol on the need for a handover and to send the relevant handover messages to the remote nodes. The HITr signal is sent less than $500 \mathrm{~ms}$ before the handover process starts.

The SMR message sent to the PXTR for the proxy router to solicit the current MN's mapping information is also sent to the loc-server. On receiving the SMR messages, the two nodes generate a map-request message to the $\mathrm{MN}$ prompting the $\mathrm{MN}$ to send back map-reply to the nodes (Fig. 2) . The map-reply message to the PXTR contains the loc-server address in the locator field of the packet but a null value in the similar field of the packet going to the loc-server. This map-reply message prompt the loc-server to listen to data packets destined to the EID contained in the message once it checks and verifies the EID from its database of accepted prefixes/addresses. The PXTR, on the other hand, uses the loc-server address in the map-reply message to forward the MN-bound packets to the loc-server during the handover process, as shown in Fig. 2, which are buffered to be sent to the MN at the 
completion of the process.

When the interface is reconfigured after the handover, similar messages are exchanged between the MN and the PXTR as well as the loc-server. The map-reply message sent to the two nodes contains the locator of the $\mathrm{MN}$ in the locator field of the packets. The only difference between the packet going to the PXTR and the one going to the server is the generated nonce. The nonce value is used in LISP to ensure that packets received are from the nodes that claim to be sending them. Consequently, all packets buffered by the loc-server are forwarded to the MN, whereas the PXTR resumes sending all received packets to the MN. It should be noted that loc-server is not involved in any subsequent data plane operations until the next HITr.

\section{Performance Evaluation}

In order to have an understanding of how DASH players perform on LISP-MN network environment with reduced packet loss as aimed with the improved LISP-MN architecture, we set up a laboratory testbed with the major components of LISP-MN ${ }^{3}$ plus the locserver implemented. The testbed consists of nine desktops PCs configured as one MN, two access routers (ARs), one map-server, one PXTR, a loc-server, one $\mathrm{CN}$ and two backbone routers. This allows us to evaluate the performance of DASH players on both the legacy LISP-MN and the proposed LISP-MN architecture, henceforth called plain_LISP and loc_LISP, respectively. Since the speed of wireless networks differ between different radio technologies, we will run tests for both fast and slow wireless links. We consider fast link to be a radio link that has more than 2.0 mbps download capacity as could be attained with Wi-Fi networks and 3/4G; while slow is considered to be a channel with less than 2.0 mbps capacity emulating low-speed Wi-Fi/cellular connectivities. For the wireless radio, 802.11 bgn antennas were used on the Ubuntu desktops serving as the MN and AR, hence the bandwidth of fast link channels can be as high as $20 \mathrm{Mbps}$.

Since it is not possible to test every player, we chose a representative player from each of the three categories, discussed in subsection 2.2, for our experiments. For the throughputbased player, we opted for the open source version of microsoft smooth streamer [2], while for the buffer-based, we chose the player proposed in [12]. Finally, we chose the player proposed in [17] as representative of hybrid players. Apache 2.4.17 was used as the web server for hosting the 'Big Buck Bunny' video dataset from [16]. It consisted of different quality levels, ranging from 50 to $5,000 \mathrm{kbps}$.

We assumed a scenario where a user walks across distinct wireless networks and makes six handovers while streaming video. We use the metrics below to evaluate the performance of the players. All of the metrics used are known to affect user quality of experience [9, 10]:

- Average quality level/video rate: measured in kbps and calculated as

$$
\frac{t 1 q 1+t 2 q 2 \ldots t n q n}{t n-t 1}
$$

where $t$ is the time duration that a particular quality level is downloaded, and $q$ is the actual quality level downloaded in the corresponding time.

- Rebuffers: the total number of video freeze per streaming session.

- Instability: the fraction of successive chunk requests by a player in which the requested video rate changes [136], measured at the steady-state.

Hence, we will look at the impact of low packet loss on video quality and buffer dynamics, network utilisation, and stability of the players. 


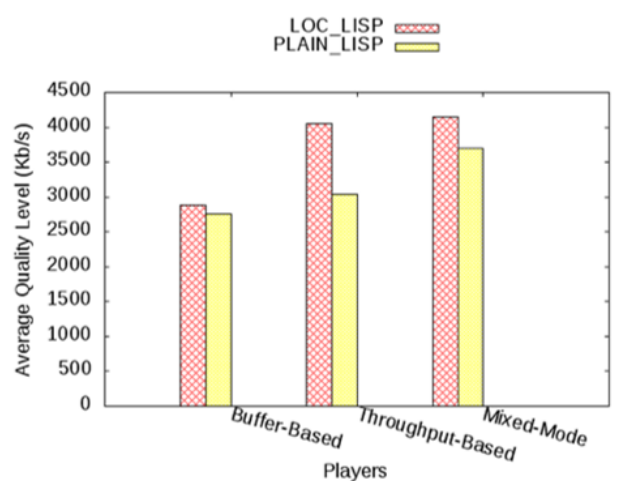

A (Fast)

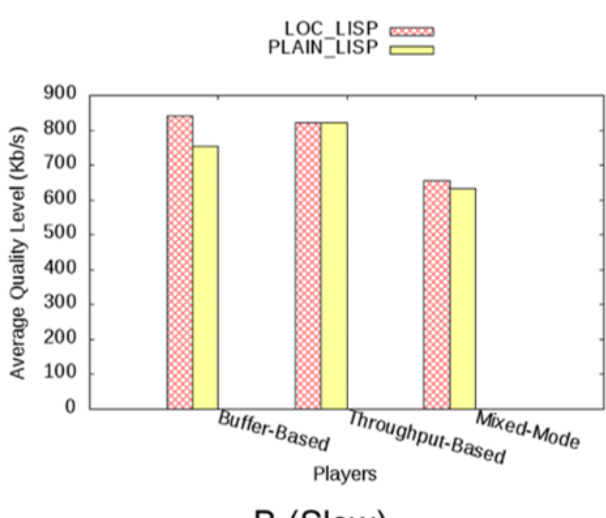

B (Slow)

Figure 3. Average Quality Level Attained by the Players on Fast and Slow Links.

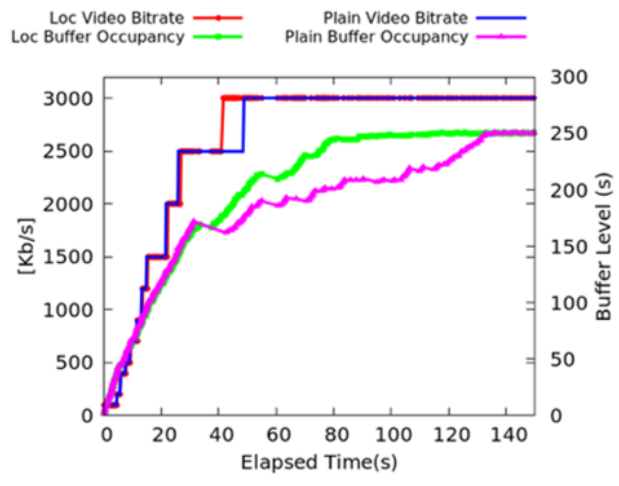

A (Fast)

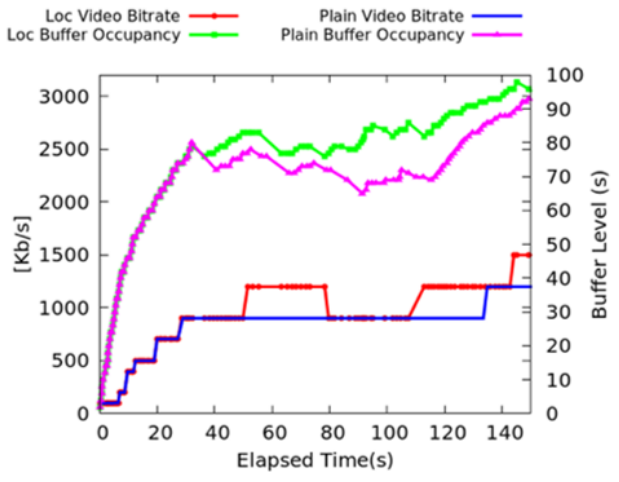

B (Slow)

Figure 4. Buffer-Based Player: Buffer and Average Quality Levels for Fast and Slow Links.

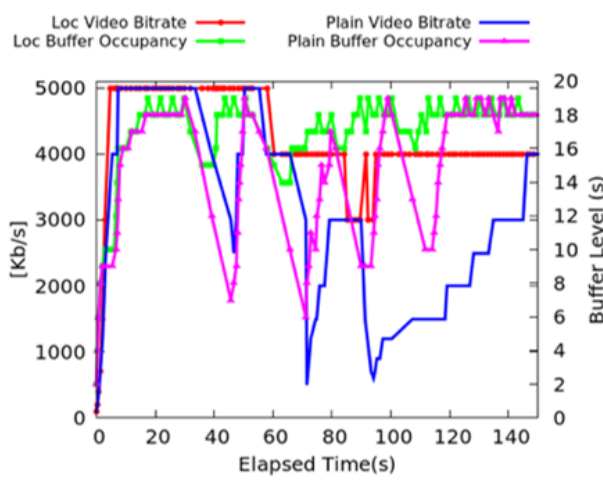

A (Fast)

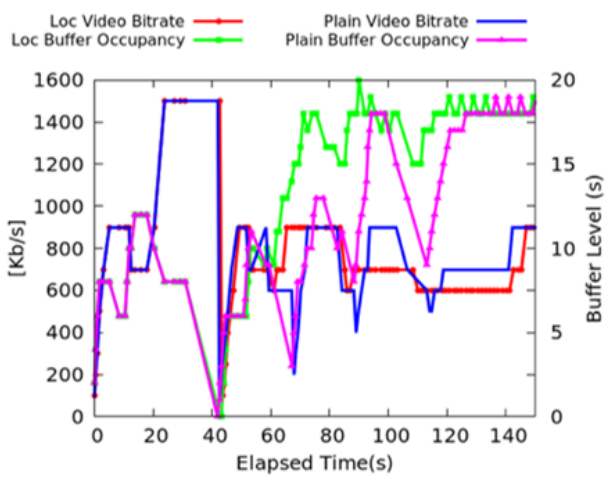

B (Slow)

Figure 5. Throughput-Based Player: Buffer and Average Quality Levels for Fast and Slow Links.

\subsection{Average Quality Level}

In Fig. 3, the average video rate over the streaming session is presented. As can be seen, all the players, when streaming using loc_LISP enjoy higher video rate in comparison to

\footnotetext{
${ }^{3}$ We used lispmob.org implementation which has since matured to open overlay router https://github.com/OpenOverlayRouter/oor/wiki
} 


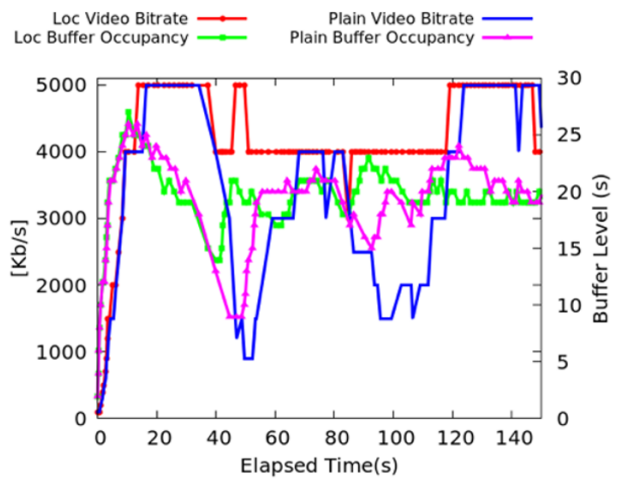

A (Fast)

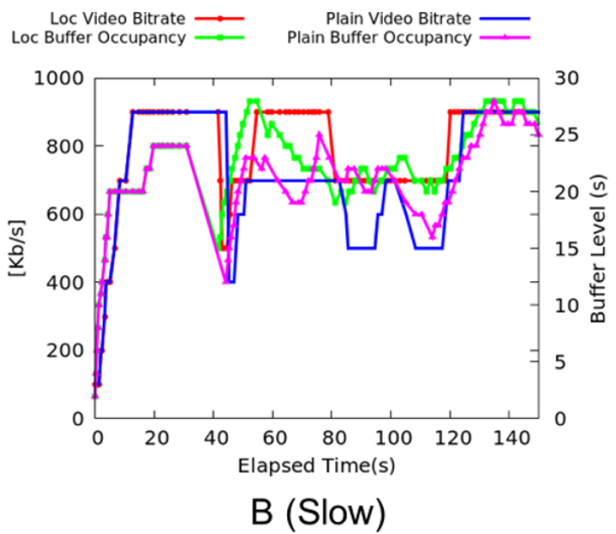

B (Slow)

Figure 6. Mixed-Mode Player: Buffer and Average Quality Levels for Fast and Slow Links.

Table 1. Network Utilisation on Fast Link.

\begin{tabular}{|c|c|c|}
\hline & \multicolumn{2}{|c|}{ Utilisation } \\
\hline Players & LOC LISP & PLAIN LISP \\
\hline Buffer-Based & 58 & 55 \\
\hline Throughput-Based & 81 & 61 \\
\hline Mixed-Mode & 83 & 74 \\
\hline
\end{tabular}

plain_LISP. This can be attributed to the reduction in SDT and packet loss that results from buffering packets by the loc-server (see results for SDT and Packet Loss in Appendix). Nonetheless, the mixed-mode player shows the best performance followed by the throughputbased player. We can also observe the improvements in the average quality levels of the different players from figures 4,5 , and 6 . There was a minimal improvement in download quality for buffer-based player running on loc_LISP as the only difference observed for the fast link (Fig. 4) was at the few seconds after the first handover ${ }^{4}$. This is because with the buffer level at around 150 seconds (s), the SDT of less than 6s is too small a time to perturb the player. We observed some improvements for the buffer-based player on slow link however, as more and more handovers were executed.

We can also notice that buffer drains faster when plain_LISP is used in comparison to loc_LISP, in all the scenarios. Again due to the shorter SDT and improved throughput performance post-handover (see throughput results in Appendix). The mixed-mode player recorded the best performance when ran on the LOC_LISP as depicted in the figures 4, 5 , and 6 , it nevertheless recorded the lowest video rate on the slow link. We can also see that the throughput-based player is the highest beneficiary of the improved architecture on the fast link with an average download video rate of 4,055 kbps against 3,042 kbps for plain_LISP; it is followed by the mixed-mode player. However, the buffer-based player shows the best improvement on loc_LISP against plain_LISP on the slow link, with $840 \mathrm{kbps}$ to $753 \mathrm{kbps}$ average quality downloaded respectively.

\subsection{Network Utilisation}

Since the available bandwidth of the fast link is greater than the highest available video rate, we calculate link utilisation using the percentage difference between the downloaded quality level and the highest available video download, which is 5,000 kbps (as stated earlier) - a player that downloads at $2000 \mathrm{kbps}$ of the $5000 \mathrm{kbps}$ video quality available is considered to be only utilising $40 \%$ of the fast link. The mixed-mode player shows the highest utilisation amongst the three players on the fast Internet connection (Table 1) with $83 \%$ for loc_LISP

${ }^{4}$ six handovers executed with 24 seconds interval 
Table 2. Network Utilisation on Slow Link.

\begin{tabular}{|c|c|c|}
\hline & \multicolumn{2}{|c|}{ Utilisation } \\
\hline Players & LOC LISP & PLAIN LISP \\
\hline Buffer-Based & 47 & 42 \\
\hline Throughput-Based & 46 & 47 \\
\hline Mixed-Mode & 36 & 35 \\
\hline
\end{tabular}
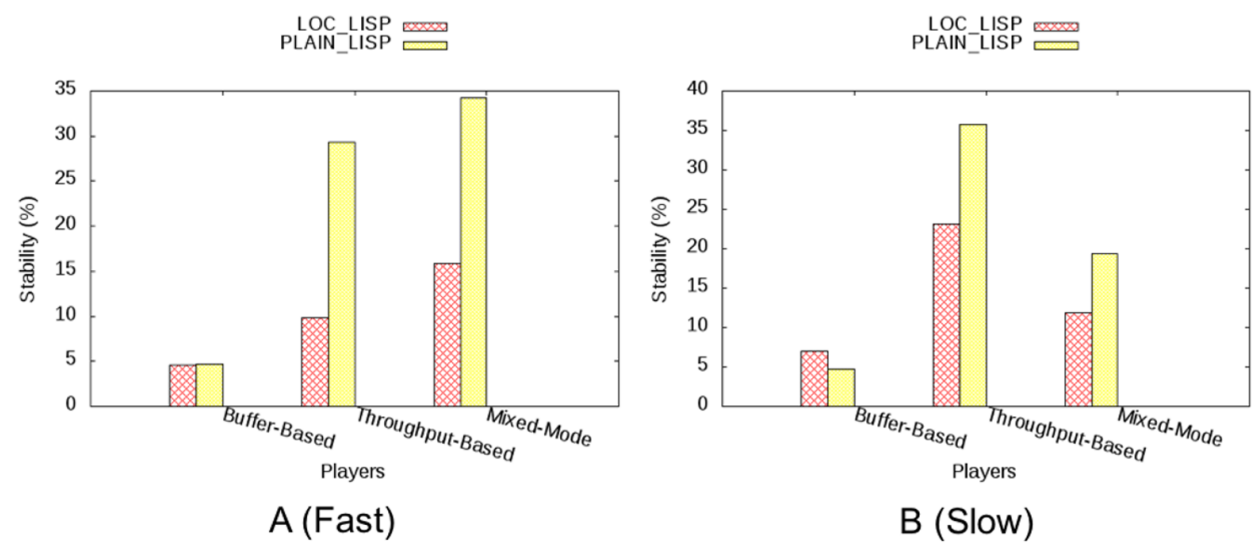

Figure 7. Stability: Percentage Change in Video Quality For Fast and Slow Links.

against 74\% for plain_LISP but performs poorly in slow networks as shown in Table 2 . The buffer-based player shows slightly better performance on loc_LISP by only $1 \%$ against throughput-based player on slow networks. The buffer-based player, however, did poorly on the fast network in comparison.

While there is a significant difference in utilisation between loc_LISP and plain_LISP on the fast link for throughput-based and mixed-mode players, the buffer-based player shows little sensitivity to the improvement in throughput and reduced SDT that comes with loc_LISP showing only $3 \%$ difference in link utilisation.

\subsection{Stability of Players}

In the event of a changing condition, a DASH player is expected to adjust its requested video rate. However, changing video quality level often is known to be detrimental to the quality of experience [6]. It is worth noting that the higher the percentage value on the stability graph (Fig. 10) the more unstable a player is. It is obvious from the figure that loc LISP produces better stability for the throughput-based and the mixed mode players within the two different links. However, there is a comparable performance, when using the fast link for the buffer-based player with plain_LISP showing better stability on the slow link. This is due to the fact that an MN can maintain a particular quality level while using a buffer-based player even amidst handovers as we saw in Fig. 4. And although receiving buffered packets at the completion of the handover improved the average video quality by $3 \%$ on the fast link and $5 \%$ on the slow link, it comes at the expense of stability.

The most significant improvement in stability recorded is that of the throughput-based player on the fast link, with the player being $67 \%$ more stable on loc_LISP than on plain_LISP, followed by the mixed-mode player. This is expected because as a player that relies on throughput, it is always expected to perform better on a network that provides significantly more of it. Hence, the improvement in throughput brought about by the introduction of loc-server helps the player reaches higher stability levels despite the many handovers during streaming. The mixed-mode player shows better stability than the throughput-based player on a slow link with plain_LISP showing higher instability for both players in comparison to 
loc_LISP.

\section{Discussion}

The three different players chosen for the evaluation have all demonstrated improved performance when running on top of loc_LISP with the mixed-mode player showing the best performance on the fast wireless link. The throughput-based player recording the highest download rate of the three is not surprising as the new architecture improved the throughput experience during handover. The improvement in throughput is also the reason why buffer draining is significantly reduced when the players were run on top of the new architecture.

We have also observed improvements in network utilisation on the fast link with the players running on loc_LISP utilising the available network capacity to download higher video rates. Loc_LISP did not record any remarkable improvement against the Plain_LISP in network utilisation on the slow link, especially for the throughput-based player. This is generally because with limited download capacity, the players maintain the current quality level despite sensing improvement in throughput and a reduction in SDT, as the next higher quality level could not be sustained with the available network resources from the client's perspectives.

\section{Related Work}

In addition to LISP-MN, a number of Loc/ID protocols have been proposed with the aim of supporting mobility and improving applications performance on the Internet. These include Internet Vastly Improved Plumbing (IVIP), Identifier Locator Network Protocol (ILNP) and Host Identity Protocol (HIP).

\section{Internet Vastly Improved Plumbing (IVIP)}

IVIP [22] proposes the separation of the core network from the edge (Identifier space) by providing two namespaces (addresses). The core address space is used in the default free zone while a block of addresses is used in the edge networks with the relationship between the two blocks maintained by a query server database. A translating tunnel router (TTR) is assigned a core address to serve as the anchor point and an ETR for the MN. The MN, on the other hand, is assigned an address from the edge network address space. All MN's communications are achieved via the TTR. Only the MN's CoA changes when the MN moves and the TTR resumes communication as soon as the MN updates its location.

\section{Identifier Locator Network Protocol (ILNP)}

ILNP [3] proposes the replacement of the 128 bits IPv6 address with two distinct namespaces of 64 bits each, network locator (L64) and node identifier (NID) with DNS as the rendezvous server (RVS) to provide the mapping between the two namespaces. The L64 is analogous to the address prefix for routing in IPv6. Data sessions are formed using only the NID with the L64 used for routing of packets. Nodes achieve mobility by changing the L64 when they move and update their record with the DNS accordingly.

\section{Host Identity Protocol (HIP)}

HIP [18] introduces a host identity namespace, for naming IP nodes with a statically globally unique host identifier in form of a cryptographic key called a host identity tag (HIT). HIT is in the form of an IPv6 address and used by the transport and upper layers of the OSI network stack for communication sessions. The IP address at the network layer, the locator, 
is used for routing purposes. The mapping between the HITs and IP addresses on the Internet is provided using an RVS, such as the DNS. HIP data session begins with the base exchange process to ensure the communicating nodes have relevant cryptographic keys to use in the session. The MN updates the remote nodes of its movement by sending the HIP update message to ensure that its packets are delivered to its new location.

All the four Loc/ID protocols experience some delay during handover because, in addition to link layer handover processes, each protocol has a defined signalling message necessary to switch an MN's traffic to a new link. These messages include updating PXTR and map-server (and CNs in some scenarios) on LISP-MN, TTR on IVIP, and DNS together with CNs on ILNP and HIP. This delay in handover causes the packets sent at the start of a handover to be dropped by the previous router. And for some connectionless data sessions, the $\mathrm{CN}$ may continuously send packets to the $\mathrm{MN}$ oblivious of its short downtime during the handover process.

Our newly improved architecture, as demonstrated with LISP-MN, ensures that packets sent during handover are saved by the loc-server and forwarded to the receiving $\mathrm{MN}$ upon handover completion. This was shown, in section 4 , to significantly improve video performance on an MN running LISP-MN protocol. The loc-server solution could be deployed to work with the other Loc/ID protocols highlighted.

\section{Conclusion}

In this paper, we introduced an improved LISP-MN architecture aimed at improving the performance of mobile video streaming services. A new node called loc-server is added to the LISP-MN architecture. This node is tasked with buffering incoming packets during handover and forwarding the packets to the $\mathrm{MN}$ on the completion of the handover process. We implemented the proposed architecture on a laboratory testbed. We evaluated the performance of a number of MPEG-DASH compliant players over the improved LISP-MN architecture in comparison to vanilla LISP-MN. Results show that a reduction in packet loss and service disruption time during handover can be achieved when the proposed architecture is employed. These reductions have been shown to significantly improve MPEG-DASH players' performance. 


\section{References}

[1] 2014. Dynamic adaptive streaming over http (dash) - part 1: Media presentation description and segment formats. International Standard ISO/IEC 23009-1:2014 Second Edition .

[2] 2014. Slextensions adaptive streaming. URL: https://slextensions. svn. codeplex.com/svn/trunk/SLExtensions/AdaptiveStreaming. accessed: March, 2014.

[3] Atkinson, R.J., Bhatti, S.N., 2012. Identifier-Locator Network Protocol (ILNP) Architectural Description. Technical Report. Internet Research Task Force (IRTF) Request for Comments: 6740.

[4] Biernacki, A., Tutschku, K., 2013. Performance of http video streaming under different network conditions. Multimedia Tools and Applications 72, 1143-1166.

[5] CVN, 2016. Cisco Visual Networking Index: Global Mobile Data Traffic Forecast Update, 2015-2020 White Paper. Technical Report. Cisco Systems Inc.

[6] Dobrian, F., Sekar, V., Awan, A., Stoica, I., Joseph, D., Ganjam, A., Zhan, J., Zhang, H., 2011. Understanding the impact of video quality on user engagement. SIGCOMM Comput. Commun. Rev. 41, 362-373. URL: http://doi.acm.org/10.1145/ 2043164.2018478.

[7] Farinacci, D., Fuller, V., Meyer, D., Lewis, D., Jan. 2013. Locator/id separation protocol (lisp). IETF, RFC 6830 .

[8] Farinacci, D., Lewis, D., Meyer, D., White, C., 2021. LISP Mobile Node. Internet-Draft draft-ietf-lisp-mn-10. Internet Engineering Task Force. URL: https://datatracker.ietf.org/doc/html/draft-ietf-lisp-mn-10. work in Progress.

[9] Garcia, M.N., De Simone, F., Tavakoli, S., Staelens, N., Egger, S., Brunnström, K., Raake, A., 2014. Quality of experience and http adaptive streaming: A review of subjective studies, in: Quality of Multimedia Experience (QoMEX), 2014 Sixth International Workshop on, IEEE. pp. 141-146.

[10] Georgopoulos, P., Elkhatib, Y., Broadbent, M., Mu, M., Race, N., 2013. Towards network-wide qoe fairness using openflow-assisted adaptive video streaming, in: Proceedings of the 2013 ACM SIGCOMM Workshop on Future Human-centric Multimedia Networking, ACM, New York, NY, USA. pp. 15-20. URL: http: //doi.acm.org/10.1145/2491172.2491181, doi:.

[11] Gorius, M., Yongtao, S., Herfet, T., 2012. Dynamic media streaming over wireless and mobile ip networks, in: Consumer Electronics - Berlin (ICCE-Berlin), 2012 IEEE International Conference on, pp. 158-162.

[12] Huang, T.Y., Johari, R., McKeown, N., 2013. Downton abbey without the hiccups: Buffer-based rate adaptation for http video streaming, in: Proceedings of the 2013 ACM SIGCOMM workshop on Future human-centric multimedia networking, ACM. pp. 9-14.

[13] Isah, M., Edwards, C., 2015. Inter-domain mobility with lisp-mn - a performance comparison with mipv6, in: 2015 8th IFIP Wireless and Mobile Networking Conference (WMNC), pp. 80-87. 
[14] Isah, M., Simpson, S., Edwards, C., 2018. An improved lisp mobile node architecture. Journal of Network and Computer Applications 118, 29 - 43. URL: http://www. sciencedirect.com/science/article/pii/S108480451830105X.

[15] Kopp, W., Klein, A., 2011. Locator/identifier split, in: Carle, G., Schmitt, C. (Eds.), Proceedings of the Seminars Future Internet (FI) and Innovative Internet Technologies and Mobile Communications (IITM), Munich.

[16] Lederer, S., Müller, C., Timmerer, C., 2012. Dynamic adaptive streaming over http dataset, in: Proceedings of the 3rd Multimedia Systems Conference, ACM, New York, NY, USA. pp. 89-94. URL: http://doi.acm.org/10.1145/2155555.2155570.

[17] Miller, K., Quacchio, E., Gennari, G., Wolisz, A., 2012. Adaptation algorithm for adaptive streaming over http, in: 2012 19th International Packet Video Workshop (PV), pp. 173-178.

[18] Moskowitz, R., Nikander, P., 2006. Host identity protocol (hip) architecture. IETF RFC 4423.

[19] Quoitin, B., Iannone, L., De Launois, C., Bonaventure, O., 2007. Evaluating the benefits of the locator/identifier separation, in: Proceedings of 2nd ACM/IEEE international workshop on Mobility in the evolving internet architecture, ACM. p. 5.

[20] Sani, Y., Isah, M., Edwards, C., Mauthe, A., 2017. Experimental evaluation of the impact of mobility management protocols on http adaptive streaming. IET Networks $6,186-192$.

[21] Sani, Y., Mauthe, A., Edwards, C., 2015. Modelling video rate evolution in adaptive bitrate selection, in: 2015 IEEE International Symposium on Multimedia (ISM), IEEE. pp. 89-94.

[22] Whittle, R., Russert, S., 2008. Ttr mobility extensions for coreedge separation solutions to the internet's routing scaling problem. http://www.firstpr.com.au/ip/ivip/TTR-Mobility.pdf. 


\section{Appendix}

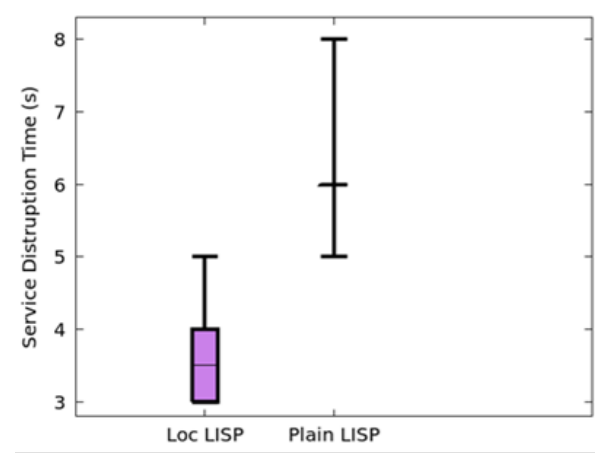

A

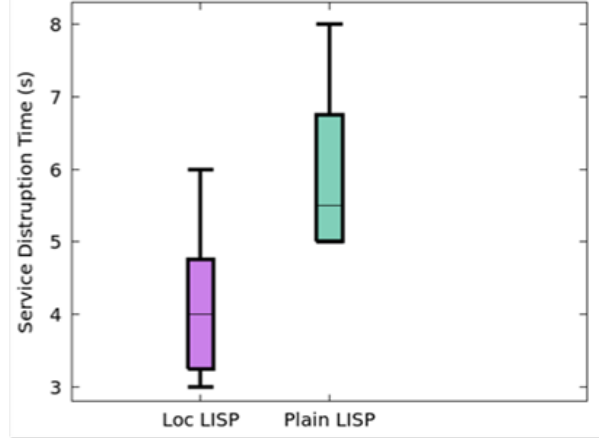

B

Figure 8. TCP Service Disruption Time for Fast (A) and Slow (B) Links.
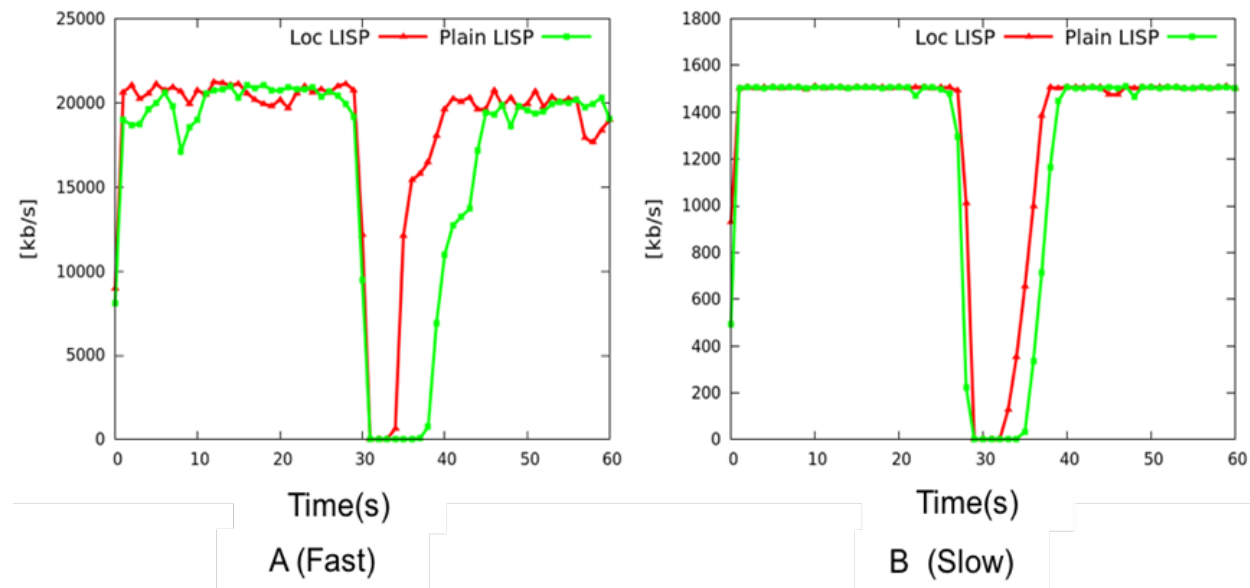

Figure 9. TCP Throughput 
LOC_LISP $\rightleftarrows \infty$

PLAIN_LISP

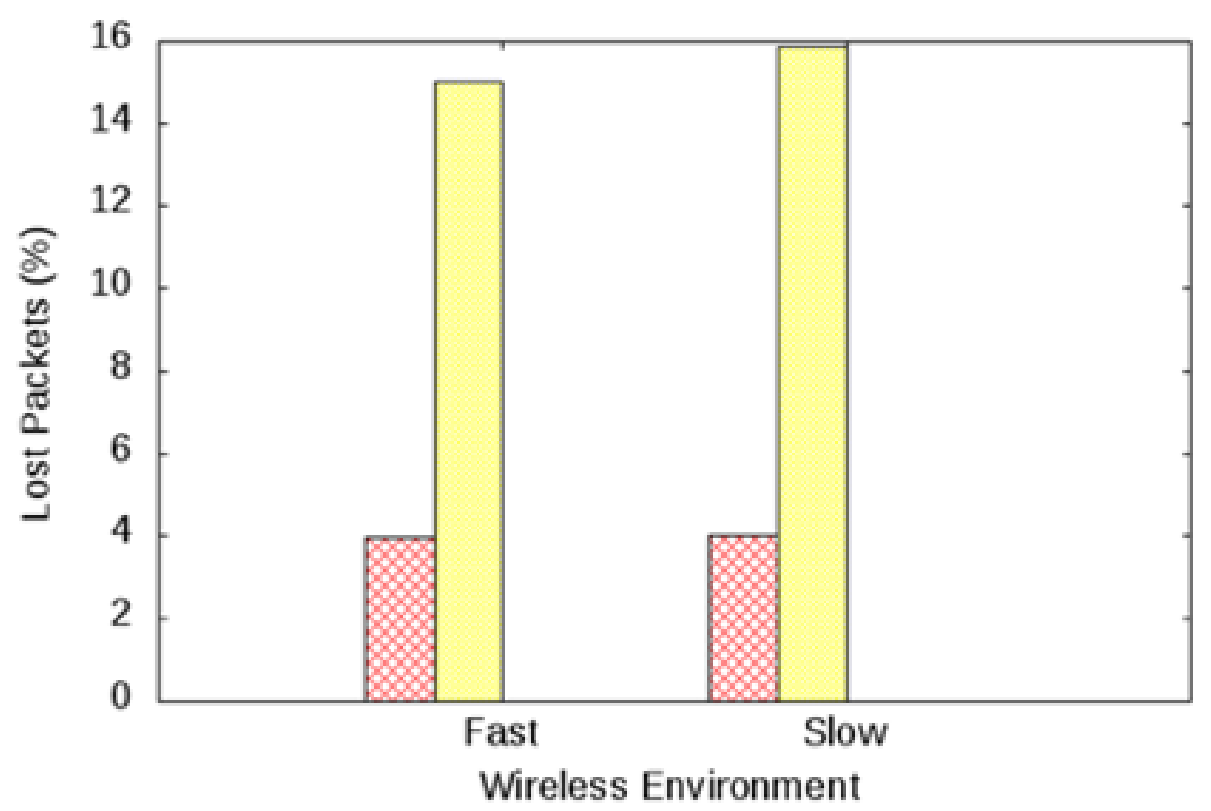

Figure 10. Packet Loss During Handover. 